\title{
Polyamphoteric flocculants for the enhanced separation of cellular suspensions
}

Kathryn L. Morrissey, Chunlin He, Rebeccah Z. Chapman, Lucjan Żotnierowski, and Mark P. Stoykovich*

K. L. Morrissey, C. He, R. Z. Chapman, L. Żołnierowski, Prof. M. P. Stoykovich

Department of Chemical and Biological Engineering

University of Colorado at Boulder

Boulder, CO 80309 (USA)

Email: mark.stoykovich@ colorado.edu

\begin{abstract}
:
The efficient concentration and separation of microorganisms from dilute culture suspensions is crucial to the success and productivity of many biotechnological processes. This article presents the design and characterization of polyamphoteric flocculants with a tunable, zwitterionic character for the enhanced separation of biocolloidal suspensions of yeast, wastewater, microalgae, and potentially other cellular systems. The polyamphoteric flocculants have overall molecular charges dependent upon the system $\mathrm{pH}$, thereby providing a strong electrostatic attraction to the diverse but predominantly negatively-charged cellular surfaces of the biological suspensions. The polyamphoteric flocculants with tailored charge character are shown to have higher flocculation efficiencies than comparable cationic polyelectrolytes, and have an enhanced ability to 1) adsorb to the diverse range of charge character in cellular suspensions, 2) operate over an extended range of suspension $\mathrm{pHs}$, and 3) operate at lower flocculant concentrations. These enhanced flocculation properties are shown to arise, perhaps counterintuitively, due to interactions between the negatively-charged functionality on the flocculant and the predominantly negatively-charged biocolloids.
\end{abstract}

Keywords: Polyampholyte; flocculation; Chlorella vulgaris; Saccharomyces cerevisiae; wastewater 


\section{Introduction:}

Microorganisms are used in biotechnological processes to generate diverse products including antibiotics and proteins, renewable fuels or chemicals from biomass, and ferments. These products are either emitted to the suspending medium or, in many instances, contained within the cells themselves such that efficient concentration of the microorganisms from the dilute suspensions in which they are cultured is required [1-9]. For example, biopharmaceuticals such as recombinant therapeutic proteins are commercially produced from cell cultures and fermentation processes, and following cell disruption, residual cellular debris must be removed and the protein solution further clarified to ensure product safety [10,11]. Similarly, water purification and wastewater treatment processes rely on solid/liquid separations to remove suspended solids, cellular components, and organic materials [12,13], as well as to obtain highquality effluents for activated sludge processes [8,9]. Another commercial-scale example of the separation of dilute cellular and biocolloidal materials is microalgae harvesting for nutraceuticals or biofuels production $[3,14,15]$.

The natural sedimentation of cells and bioparticulates is prohibitively slow due to the preference of cells to avoid aggregation and to remain suspended in solution $[1,9,16,17]$. The repulsive interactions between like-charged particles in solution described by DLVO (DerjaguinLandau-Verway-Overbeek) theory $[18,19]$ and structural forces occurring at suspension interfaces contribute to the stability of the suspension $[1,9,14,17,20,21]$. The conventional separation techniques of centrifugation and microfiltration, while commonly implemented in biotechnological processes, are challenging for cellular suspensions due to the broad range of particle sizes that must be processed [10,22,23] and issues associated with biofouling [22-28]. The formation of smaller particulates in cellular solutions adds further complications and may be 
attributed to low cell viability that causes the cells to lyse [29] or overexposure of the biomass to shear (e.g., in cross flow membrane filtration) [24-26].

On the other hand, flocculation or coagulation provides an attractive approach to separate and aggregate cellular or biocolloidal suspensions, possessing the ability to increase particle size and consequently the rate of sedimentation. Typically flocculation has been engineered to occur through attractive electrostatic interactions between the flocculant and the oppositely-charged colloidal particles, which leads to particulate aggregation through mechanisms such as bridging [30,31] and charge patch neutralization [32,33]. The aggregates of particles destabilize the suspension and then rapidly sediment from solution [1,21,34-37]. Cationic or anionic polyelectrolytes have been thoroughly explored over a wide range of molecular weights, charge densities, and molecular structures for the flocculation of solely negatively- or positively-charged colloidal particles, respectively, for applications in precious metal or silica recovery, water purification, or paper pulp processing [30,31]. Cell surfaces, in comparison, display a diverse range of charge character, with a species dependent set of negatively- (usually dominant) and positively-charged surface functionality at natural culture conditions. Charge at the cell surface originates from the ionization of acid-base functional groups (most commonly carboxylate, phosphate, hydroxyl, and amine functionalities) [17,21,34,38,39], and the magnitude of the surface charge is a function of the species, $\mathrm{pH}$ and ionic strength of the culture medium, and other environmental factors [17,40-42]. Synechococcus-type unicellular autotrophic picocyanobacteria, for example, display a relatively balanced distribution of charged carboxylate (37-44 mol\%), amine (29-36 mol\%), and phosphate (26-27 mol\%) groups on the cell surface [34]. 
This article reports the ability of model polyamphoteric copolymer flocculants (i.e., polymers containing both positively- and negatively-charged components) to interact more effectively with the diverse surface charges present in biocolloidal cellular suspensions, thereby achieving higher flocculation efficiencies over a wider range of operating conditions as compared to polyelectrolyte flocculants that are only positively-charged. Polyampholytes have been shown to effectively destabilize and flocculate a variety of suspensions (e.g., fibrous pulps [43], dyes [44,45], kaolin [46], polystyrene latex [30]) and have demonstrated superior separation properties when compared to cationic and anionic flocculants $[44,45]$ likely due to their unusual interfacial properties, intra- and intermolecular Coulombic interactions, and molecular conformations [30,44,46]. Nevertheless, there is a limited molecular-level understanding of the complex mechanisms by which polyamphoteric flocculants operate and, therefore, such materials have not been applied to the flocculation of biological suspensions with diverse surface charges. Here we investigate the favorable interactions of polyampholytes with both positively- and negatively-charged surface groups exhibited by a broad range of important cellular suspensions: the fresh-water microalgae species Chlorella vulgaris [17,47], the yeast Saccharomyces cerevisiae which is one of the most exploited microorganisms in fermentable and pharmaceutical processes $[7,29]$, and wastewater sludge $[12,13]$. Note that the interaction of the flocculants with specific small molecule or biomacromolecule products in these cell cultures are not considered here, although such diverse components are inherently present in such systems. We show that polyamphoteric flocculants with tailored charge character achieve flocculation efficiencies greater than comparable cationic polyelectrolyte flocculants, and have an enhanced ability to 1) adsorb to the diverse range of charge character in cellular suspensions, 2) operate over an extended range of system $\mathrm{pH}$ values, and 3) operate at lower flocculant concentrations. 
A charge-stabilized colloidal suspension of silica microparticles with only negative surface charges is considered as a reference, and is used to elucidate the important electrostatic mechanisms by which polyampholytes flocculate cellular systems.

\section{Materials and Methods:}

\section{Synthesis of amphoteric flocculants [48,49]}

Materials: $N, N$-dimethylaminopropyl acrylamide (DMAPAA) (98\%, light yellow oil), ammonium persulfate, and acetone were purchased from Fisher Scientific. 3-acrylamidopropyltrimethyl-ammonium chloride solution (DMAPAA-Q) (98\%, light pink oil) and acrylic acid (99\%) were purchased from Sigma Aldrich. Prior to polymer synthesis, the DMAPAA and acrylic acid monomers were purified by vacuum distillation and the DMAPAA-Q monomer was purified over basic alumina adsorption columns to remove inhibitor, while the ammonium persulfate was used as received.

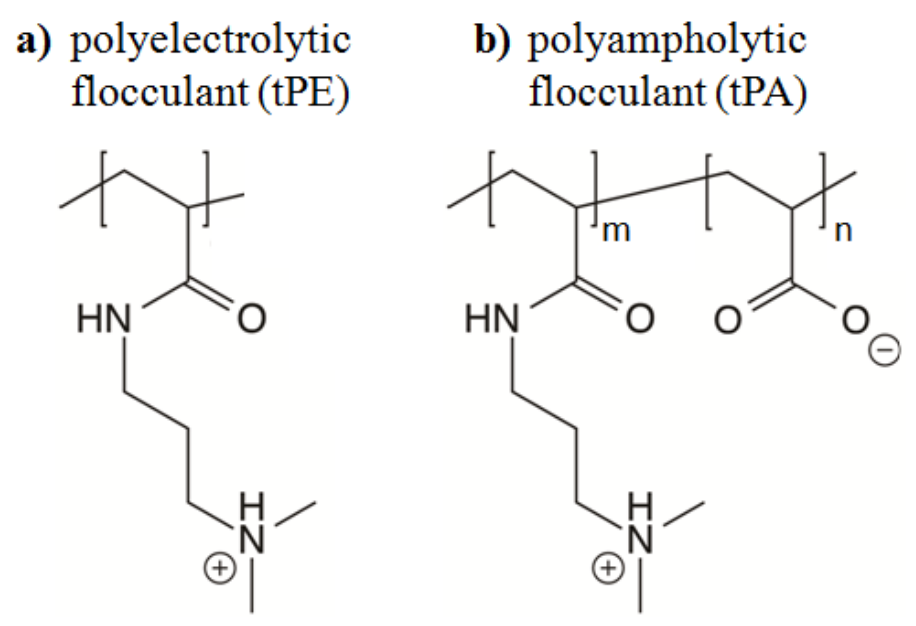

Scheme 1. a) Cationic polyelectrolyte (tPE) flocculant product from the tertiary amine DMAPAA monomer at neutral $\mathrm{pH}$. b) Polyampholyte (tPA) flocculant with $75 \mathrm{~mol} \%$ DMAPAA and $25 \mathrm{~mol} \%$ acrylic acid repeat units has a zwitterionic character at neutral $\mathrm{pH}$. 
Briefly, the free-radical polymerization of the copolymer flocculants involved dissolving the $\left(\mathrm{NH}_{4}\right)_{2} \mathrm{~S}_{2} \mathrm{O}_{8}$ initiator and monomers in deionized water $\left(\mathrm{DI} \mathrm{H}_{2} \mathrm{O}\right)$ followed by heating at $60-70^{\circ} \mathrm{C}$ and stirring for $48 \mathrm{hrs}$. For the synthesis of the flocculants with the quaternary amine DMAPAA-Q monomer, the reaction mixture was adjusted to a $\mathrm{pH}$ of 4 with $1 \mathrm{M} \mathrm{NaOH}$ prior to polymerization. All other polymer reaction mixtures were polymerized at their natural $\mathrm{pH}$. The viscous reaction mixture was then precipitated into acetone to recover and purify the polymer

product. The composition of each product was determined by ${ }^{1} \mathrm{H}-\mathrm{NMR}$ in DMSO- $d_{6}$ at room temperature. Aqueous gel permeation chromatography with a Waters 515 HPLC pump and Waters 2414 refractive index detector (Waters Corporation, Milford, MA) was used to determine the molecular weight distribution of the synthesized polymers. The yield and molecular characteristics of the polymer flocculants that were synthesized are listed in Table 1.

\section{Titration of polymer flocculants}

Polymer flocculant solutions in deionized water were made at a concentration of $0.25-2.5 \mathrm{wt} \%$. The solutions were adjusted to an initial $\mathrm{pH}$ of $\sim 2$ with $2 \mathrm{M} \mathrm{HCl}$ and then titrated with concentrated $\mathrm{NaOH}(0.01-10 \mathrm{M})$ to a final $\mathrm{pH}$ of at least 12 . The $\mathrm{pH}$ values and volumes were recorded using a pH meter (Five Easy FE20 and IE409 probe, Mettler Toledo) and burette, respectively.

\section{Zeta potential measurements}

Electrophoretic mobilities were measured at $22^{\circ} \mathrm{C}$ by laser Doppler micro-electrophoresis using a ZetaPALS Zeta Potential Analyzer (Brookhaven Instruments). Samples of the polymer flocculant and cellular suspensions were adjusted with $1 \mathrm{M} \mathrm{HCl}$ or $1 \mathrm{M} \mathrm{NaOH}$ to achieve a range 
of solution pHs from 0-14 and then diluted in a 1:15 ratio with $1 \mathrm{M} \mathrm{KCl}$. Ten measurements were completed per sample, from which the average and standard deviation were determined. Conversions to $\zeta$ were made using the Smoluchowski equation.

\section{Culturing of Chlorella vulgaris [49-51]}

C. vulgaris, strain UTEX 395, was grown in glass photobioreactors with modified Bold Basal media (MBBM) and external illumination from fluorescent bulbs (32 W, 4000K color). Aeration and agitation was supplied by bubbling with house air (pressure of 65 psi, and filtered with a $0.01 \mu \mathrm{m}$ coalescing prefilter and $1 \mu \mathrm{m}$ particulate filter). Optical density (OD) measurements at a wavelength of $750 \mathrm{~nm}$ (Agilent 8453 series UV-visible spectrophotometer; Agilent Technologies, Santa Clara, CA) were used to determine the concentration and turbidity of the algal cultures

\section{Culturing of Saccharomyces cerevisiae}

S. cerevisiae, strain BY 4741, was grown in yeast extract-peptone-dextrose (YEPD) medium with 2 vol \% additional dextrose in $500 \mathrm{~mL}$ Erlenmeyer flasks with external illumination from fluorescent bulbs $(32 \mathrm{~W}, 4000 \mathrm{~K}$ color) on a shaker plate (Henry Troemner, LLC). Yeast suspensions were washed three times and resuspended in $\mathrm{DI} \mathrm{H}_{2} \mathrm{O}$. The concentration and turbidity of the yeast cultures were determined by OD measurements at a wavelength of $600 \mathrm{~nm}$.

\section{Preparation of wastewater sludge suspensions}

Anaerobically digested wastewater sludge was obtained from the Boulder Wastewater Treatment Plant (Boulder, CO). The sludge was diluted 4-fold with $\mathrm{DI} \mathrm{H}_{2} \mathrm{O}$ in preparation for flocculation. 
The concentration and turbidity of the wastewater suspension was determined by OD measurements at a wavelength of $750 \mathrm{~nm}$.

\section{Preparation of silica particle suspensions}

Negatively-charged silicon dioxide particles $(0.5-10 \mu \mathrm{m}$ diameter) were purchased from Sigma Aldrich and dissolved in $\mathrm{DI} \mathrm{H}_{2} \mathrm{O}$ at a concentration of $0.5 \%$ w/w. A stable suspension was achieved upon vortexing and sonication for $1 \mathrm{hr}$. The concentration and turbidity of the silica suspension was determined by OD measurements at a wavelength of $750 \mathrm{~nm}$.

\section{8. pH-dependent flocculation of cellular suspensions [49,52]}

The procedure used to characterize flocculation behavior over a wide range of $\mathrm{pH}$ values involved the addition of the polymer flocculants (in ppm) to the colloidal suspensions, vortexing for $30 \mathrm{~s}$ to achieve complete mixing, and gravitational settling for a fixed period of time. After settling, $200 \mu \mathrm{L}$ of the supernatant was removed for OD measurements (using a 1:5 dilution with culture media for C. vulgaris, S. cerevisiae, and wastewater sludge, and a 1:8 dilution with $\mathrm{H}_{2} \mathrm{O}$ for silica). To eliminate concentration effects on the flocculation process and efficiency, the concentrations of the suspensions were adjusted prior to flocculation; algal and wastewater sludge suspensions were adjusted to an $\mathrm{OD}_{750 \mathrm{~nm}}$ of 5 , yeast cultures were adjusted to an $\mathrm{OD}_{600 \mathrm{~nm}}$ of 5 , and silica suspensions were prepared at an $\mathrm{OD}_{750 \mathrm{~nm}}$ of 8 . Sets of $1 \% \mathrm{w} / \mathrm{w}$ flocculant solutions were prepared by dissolution into DI $\mathrm{H}_{2} \mathrm{O}$ previously adjusted with $1 \mathrm{M} \mathrm{HCl}$ or $1 \mathrm{M}$ $\mathrm{NaOH}$ to achieve a range of solution $\mathrm{pHs}$ from 0-14. Aliquots of C. vulgaris, S. cerevisiae, wastewater sludge, and silica suspensions were adjusted with $1 \mathrm{M} \mathrm{HCl}$ or $1 \mathrm{M} \mathrm{NaOH}$ to achieve a range of suspension $\mathrm{pHs}$ from 0-14. The flocculant efficiency at different $\mathrm{pHs}$ were 
characterized on $10 \mathrm{~mL}$ (microalgae, wastewater, and silica) or $4 \mathrm{~mL}$ (yeast) samples of the different suspensions at an efficient flocculant operating concentration, which was defined for each suspension type as the lowest flocculant dosage necessary to achieve flocculation efficiencies of $>95 \%$. In all cases, the $\mathrm{pH}$ of the flocculant was selected to match the $\mathrm{pH}$ of the suspension to which it was applied. All flocculation experiments were performed in triplicate with appropriate controls in place.

\section{Results and Discussion:}

Polyelectrolytic and polyampholytic flocculants were polymerized (Scheme 1) from the monomers $N, N$-dimethylaminopropyl acrylamide (DMAPAA) and acrylic acid [49,51]. The polyelectrolyte flocculant (tPE, Table 1) contains only the positively-charged DMAPAA repeat unit, which is a tertiary amine such that at low to moderate solution pHs it becomes a cationic homopolymer (100 mol\% positive, $0 \mathrm{~mol} \%$ negative) designed to flocculate negatively-charged suspensions. With the ability to adopt varying molecular charges as provided by the positivelycharged (75 mol\% DMAPAA repeat unit) and negatively-charged (25 mol\% acrylic acid repeat unit) functionalities, the polyamphoteric flocculant (tPA, Table 1) is designed to efficiently clarify cellular suspensions with diverse surface charges. The primary role of the positive functionality is to bind to and to aggregate the predominantly negatively-charged cells leading to flocculation, while the inclusion of functional groups on the flocculants that can become negatively-charged under tunable and switchable conditions enables the flocculant to be additionally attracted to the minority positive charge groups. The composition and charge ratio in such polyamphoteric flocculants may be easily controlled by varying the monomer feed ratio during the copolymerization synthesis (see Ref. [49] for relevant examples and compositions. 
Anionic polyelectrolyte copolymers with acrylic acid functionalities also may be synthesized using these methods or reversible addition-fragmentation chain-transfer polymerization [53] and serve as effective flocculating agents for positively-charged suspensions, but such materials are not considered here given the predominance of negatively-charged surfaces in cellular systems.

With the fractional charge of the tPE and tPA polymer flocculants dependent on system $\mathrm{pH}$, an overall positive charge dominates at low $\mathrm{pH}$ and overall neutrality (tPE) or negative charge (tPA) becomes activated at higher $\mathrm{pH}$. The $\mathrm{pKa}$ values of the charged components were determined using titration curves (Fig. 1a) of each flocculant and the resulting first derivative (insert in Fig 1a.). It was found that the positive DMAPAA-functionality has a pKa of $8.9 \pm 0.3$ and the negative acid-functionality has a $\mathrm{pKa}$ of $3.7 \pm 0.1$ [49]. Figure $1 \mathrm{~b}$ provides the fractional charge on the polyelectrolytic and polyampholytic flocculants as a function of system $\mathrm{pH}$, using the experimentally measured pKa values of the flocculant components. Similarly, Figure 1c presents zeta potential values of both flocculants as a function of $\mathrm{pH}$, emphasizing the deprotonation that each flocculant undergoes as $\mathrm{pH}$ is increased. The cationic tPE flocculant has a zeta potential value of $\sim 0 \mathrm{mV}$ at a $\mathrm{pH}$ of 12 , indicating that a neutral state was reached at high $\mathrm{pH}$, while the polyamphoteric tPA flocculant becomes net negatively-charged at a $\mathrm{pH}$ of 12 with a zeta potential value of $-21 \pm 3 \mathrm{mV}$. The transitions in net flocculant charge at the pKa values of the positive and negative components are clearly demonstrated by both the calculated fractional charge curves and the experimental zeta potential measurements, albeit with subtle differences at intermediate charged states that are thought to arise due to aggregation of the flocculants.

The $\mathrm{pH}$ of biocolloidal suspensions to which flocculants are applied may influence the protonation and/or deprotonation state of the charged components of the flocculants and thus 
their flocculation efficiency for the separation. The $\mathrm{pH}$-tunable nature of the flocculant charge is reported in Figure 2 by examining the dependence of flocculation efficiency on the overall culture $\mathrm{pH}$ for the recovery of the microalgae $C$. vulgaris, a favored candidate for biofuels production [17,47]. In Figure 2a, the flocculation behavior of $C$. vulgaris using the tPE and tPA flocculants is shown for culture $\mathrm{pHs}$ of $6.5,8$, and 9.5. A detailed quantitative characterization of flocculation efficiency (defined here as the ratio of the difference in optical densities (OD) of the standard control and the flocculated sample relative to the standard control, i.e., $\left.\left[1-\left(\mathrm{OD}_{750, \text { flocculated }} / \mathrm{OD}_{750, \text { standard }}\right)\right]\right)$ is also provided in Figure $2 \mathrm{~b}$ over a $\mathrm{pH}$ operating range of $0-14$. It is evident that there is a limited range of $\mathrm{pH}$ values (3 to 8) over which efficient flocculation is achieved by both flocculants. These $\mathrm{pH}$ values represent the activated operating range in which the DMAPAA and acrylic acid functionalities are protonated and deprotonated, respectively, and the tPA polymer is zwitterionic. In cultures with low $\mathrm{pH}$ values of 0 and 2 , the microalgae suspension is below the isoelectric point of $C$. vulgaris $(\mathrm{pI}=2.9$ [21]) and the cells exhibit an overall positive surface charge, therefore little flocculation is observed due to the repulsive interactions between the microalgal cells and the positively-charged flocculants. As the culture reaches a $\mathrm{pH}$ of $\sim 4$, the $C$. vulgaris cells become dominated by negatively-charged surface groups and both flocculants with high concentrations of the protonated amine are successful at flocculating the microalgae.

The slightly higher flocculation efficiency at a $\mathrm{pH}$ greater than 4 demonstrated by the amphoteric tPA flocculant, relative to the solely positively-charged tPE, is likely due to the activation of both charge components. As the basicity of the suspension is increased further, the microalgae suspension becomes more negatively-charged (a zeta potential of $-34 \pm 1 \mathrm{mV}$ at neutral growth conditions) and flocculation proceeds with a high separation efficiency. However, 
once a suspension $\mathrm{pH}$ of 8 is reached, the flocculation efficiency of tPE with only the tertiary amine functionality begins to decline. This decrease in flocculation efficiency is associated with the deprotonation of the tertiary amine as the suspension $\mathrm{pH}$ approaches the $\mathrm{pKa}$ of the amine ( $\mathrm{pKa}=8.9$ ), until at $\mathrm{pH} \sim 11$ the $\mathrm{PE}$ flocculant is fully deprontonated, uncharged, and incapable of flocculation. In contrast, the polyamphoteric tPA flocculant retains a flocculation efficiency of $>90 \%$ up to a $\mathrm{pH}$ of 9.5 and $70 \%$ at a $\mathrm{pH}$ of 10 . At high suspension pHs in excess of 12 , even the polyamphoteric flocculant stops functioning, suggesting that under these conditions the cationic groups on the cell surface also become deprotonated such that attractive electrostatic interactions with the negatively-charged tPA flocculant become impossible.

For suspensions in the $\mathrm{pH}$ range of 8 to 12 , it is therefore concluded that polyamphoteric flocculants provide a flocculating advantage over the cationic polyelectrolytes, which results from the presence of negative charges on the flocculant that interact with positive charges on the surface of the microalgal cells. This $\mathrm{pH}$ range is an important one for many biocolliodal and cellular suspensions as, for example, typical microalgal cultures are often basic (pHs of 8 10) and adopt a natural $\mathrm{pH}$ that is dependent upon many parameters including the stage of growth. Moreover, we have shown previously that the cationic polyelectrolytic and polyampholytic flocculants were able to remove $>98 \%$ of microalgae from standard cultures when added in concentrations of 70-150 and 40-80 ppm, respectively, and allowing for a sedimentation time of 10 min $[49,52]$. The lower dosages required by the polyampholytic tPA flocculant cannot be explained by differences in flocculant molecular weight or molecular structure (Table 1), and rather are suggested to arise from the inclusion of the negative acrylic acid group that leads to electrostatic attraction with the positively-charged functionalities that are distributed on the cell surface. Given that microalgae and other cellular systems have different distributions of surface 
functionalization and charge, it is likely that the composition of polyamphoteric flocculants may need to be optimized depending the specific cellular target.

Figure $2 \mathrm{~b}$ also details the flocculation behavior of polyelectrolytic (qPE) and polyampholytic (qPA) flocculants with quaternary amine, rather than tertiary amine, functionalities. For such flocculants, the quaternary amine is not $\mathrm{pH}$ sensitive and high flocculation efficiency is maintained over a wider range of suspension $\mathrm{pHs}$, from which it may be deduced that the deprontonation of the flocculant amines in TPE and tPA is solely responsible for the loss of activity under basic conditions. Although flocculants with quaternary amines are commonly applied as flocculants, there are applications for which they are not well suited [49] including cellular suspensions given their cytotoxicity. The cytotoxicity of the tPE and tPA materials on microalgae, for comparison, is relatively low at these flocculant dosages [49], with the literature reporting ecotoxicology limits for the $N$-dimethylaminopropyl acrylamide (DMAPAA) and acrylic acid components reported to range between 46-122 mg/L [54] and 0.1$236 \mathrm{mg} / \mathrm{L}$ [55,56], respectively.

As additional evidence of the role of amphoteric character in the flocculation of cellular suspensions, the flocculation of a suspension of microparticles with only negative surface charges was considered. The $\mathrm{pH}$ dependence of flocculation was characterized on a model system consisting of a charge-stabilized colloidal suspension of silica microparticles, chosen to mimic the size and concentration of the $C$. vulgaris suspension and exhibiting a constant charge density over the $\mathrm{pH}$ range evaluated due to its isoelectric point of 2 [57]. As shown in Figure 3, both the tPE and tPA flocculants, when applied at a concentration of $5 \mathrm{ppm}$, result in similar flocculation efficiencies over the entire operating range of system $\mathrm{pH}$. These observations on the silica system indicate that, when a mix of surface charges is not present on the suspended 
particulates, the inclusion of the negatively-charged acrylic acid in the polyamphoteric flocculant does not provide a flocculating advantage over the all cationic polyelectrolyte flocculant. A flocculant concentration of $5 \mathrm{ppm}$ is estimated to provide $2 \sim 3$ orders of magnitude higher concentrations of positive charge on the flocculant than there is negative charge on the surface of the colloidal silica microparticles [58], suggesting that both the polyampholytic and polyelectrolytic flocculants effectively cover the silica particles and that the mechanism of aggregation is the same.

The application and performance advantage of the polyamphoteric flocculants was demonstrated on additional biological systems. Suspensions of Saccharomyces cerevisiae at culture pHs of 4.5, 7, and 10.5 (Figure 4) and anaerobically digested wastewater sludge at pHs of 8-14 (Figure 5) were treated with the tPE and tPA flocculants. Visually and quantitatively Figure 4 demonstrates that the amphoteric tPA flocculant retains higher flocculation efficiencies over a wider range of system $\mathrm{pH}$, achieving $92 \%$ efficiency with a sample $\mathrm{pH}$ of 10.5 compared to $4 \%$ efficiency with the tPE flocculant. The observed results are similar to those of microalgae, which is not surprising given that the surface character of yeast cells are similar to that of microalgae. The net charge of yeast cells can be ascribed to side-chain carboxylic and amino groups of polypeptides, in conjunction with phosphate groups leading to a negative surface charge predominating the cells $[59,60]$. We have measured the zeta potential of the yeast at their natural culture $\mathrm{pH}$ and found it to be $-34 \pm 1 \mathrm{mV}$.

In contrast, Figure 5 demonstrates that the polyampholyte flocculant does not retain a significant flocculating advantage over the cationic polyelectrolyte flocculant when applied to wastewater sludge. Both flocculants are efficient at clarifying the suspension up to a $\mathrm{pH}$ of $\sim 10$, but then exhibit a decrease in flocculation performance at higher system pHs. At low suspension 
$\mathrm{pHs}$, the polyampholyte tPA does require less material to achieve high flocculation efficiency on the wastewater suspension ( 80 versus $100 \mathrm{ppm}$ for tPA and tPE, respectively), which agrees with the flocculation behavior on $C$. vulgaris and S. cerevisiae. The wastewater sludge at natural conditions exhibits an overall negative surface charge, similarly to microalgae and yeast, with a zeta potential of $-36.1 \pm 0.5$. Amuda et al. [13] showed, however, that increasing the $\mathrm{pH}$ of wastewater above a value of 9 markedly deteriorates the quality of the wastewater as negativelycharged organic contaminants can be produced. With an increase in negatively-charged contaminants, the relative diversity in surface charge groups decreases and the system begins to mimic a suspension with only negative charges like the silica microparticles. Therefore it was unsurprising that the quaternary amine functionalized flocculants (i.e., qPE and qPA) were able to maintain high separations efficiencies across this $\mathrm{pH}$ range.

\section{Conclusions:}

Amphoteric and zwitterionic polymer flocculants provide opportunities, as demonstrated here, for more flexible and efficient processes for the separation of cellular and biocolloidal suspensions. Flocculation efficiencies enhanced relative to comparable cationic polyelectrolyte flocculants may be achieved with polyamphoteric flocculants through their ability to 1 ) adsorb to the diverse range of charge character in cellular suspensions and 2) provide flocculation over an extended range of suspension $\mathrm{pH}$ values. Simultaneously, due to the molecular-level interactions adopted in the polyamphoteric systems, the enhanced separation efficiencies are achieved at lower concentrations of flocculant than required by analogous polyelectrolyte flocculants. Polyamphoteric flocculants may, in the future, be further optimized to have a charge character that matches the species dependent charges present in the biocolloidal suspensions to be 
aggregated. Likewise the composition of the polyamphoteric flocculants can be varied by selecting positively- and negatively-charged functionalities with different pKas, such that the operating range and $\mathrm{pH}$-responsiveness for flocculation can be tailored or extended to meet the needs of a specific application.

Polyamphoteric flocculants may therefore find utility in a diverse range of applications. Primary dewatering by flocculation reduces the burden on a secondary concentration technique for the harvesting of microalgae [61] and provides the cell immobilization for yeast suspensions necessary to enhance the productivity of fermentable and biopharmaceutical processes [7]. Polyamphoteric flocculants may also reduce the concentration of small cellular debris with their dual-charge functionality, thereby remedying some the challenges associated with the broad range of particle sizes and equipment fouling encountered in conventional separation techniques such as microfiltration. Although some applications may require remediation of the flocculants themselves from the solids stream with a downstream purification process, fortunately polyamphoteric flocculants with $\mathrm{pH}$-dependent charge character provide opportunities for novel process implementation. We have shown recently that the flocculant - biocolloid interactions may be inverted (i.e., become repulsive interactions) by adjustment of the system $\mathrm{pH}$ following the solids recovery, such that the flocculant can be desorbed, recovered, and recycled for future usage $[49,52]$. Not only would such functional materials reduce the cost burden for flocculation, but may also enhance downstream processes (e.g., in microalgal harvesting by improving oil recovery from the concentrated biomass and reducing equipment fouling). Finally, it may be anticipated that polyamphoteric flocculants may have similar benefits to those presented here for cellular systems when applied to other suspensions with complex charge distributions such as 
acid mine drainage, which is a colloidal system combining heavy metal ions, wastewater particulates, and fine-grained bed sediment [62].

\section{Acknowledgements}

This material is based upon work supported by the National Science Foundation Graduate Research Fellowship Program (K. M.) under Grant No. DGE 1144083. L.Z. was supported by the C2B2/NSF REU site program under Grant No. EEC-1005238. Saccharomyces cerevisiae and growth procedures were supplied by Denise G. Muhlrad and Carolyn J. Decker of the Parker

Group at the University of Colorado, Boulder. Zeta potential values were collected with the aid of Dr. David Metge at the U.S. Geological Survey Facility in Boulder, CO. Wastewater sludge was provided by the Boulder Wastewater Treatment Plant, Boulder, CO. Additionally, we thank Prof. Zhiyong J. Ren at the University of Colorado, Boulder for useful discussions. 
Table 1. Molar composition and molecular weight of polymer flocculants.

\begin{tabular}{|c|c|c|c|c|c|c|}
\hline & & \multicolumn{2}{|c|}{ Composition (mol \%)* } & & \\
\hline $\begin{array}{c}\text { Polymer } \\
\text { Label }\end{array}$ & Polymer Description & $\begin{array}{c}\text { Positive Monomer } \\
\text { (DMAPAA or } \\
\text { DMAPAA-Q) }\end{array}$ & $\begin{array}{c}\text { Negative Monomer } \\
\text { (Acrylic Acid) }\end{array}$ & $\begin{array}{c}\text { Yield } \\
(\%)\end{array}$ & $\begin{array}{c}\overline{\mathbf{M}}_{\mathbf{w}}{ }^{* *} \\
\left(\mathrm{~kg} \mathrm{~mol}^{-1}\right)\end{array}$ & PDI $^{* * *}$ \\
\hline tPE & $\begin{array}{c}\text { Polyelectrolyte with } \\
\text { tertiary amine }\end{array}$ & $100 \%$ & $0 \%$ & 70 & 1600 & 1.9 \\
\hline tPA & $\begin{array}{c}\text { Polyampholyte with } \\
\text { tertiary amine }\end{array}$ & $75 \%$ & $25 \%$ & 53 & 690 & 1.4 \\
\hline qPE & $\begin{array}{c}\text { Polyelectrolyte with } \\
\text { quaternary amine }\end{array}$ & $100 \%$ & $0 \%$ & 80 & 28 & 1.3 \\
\hline qPA & $\begin{array}{c}\text { Polyampholyte with } \\
\text { quaternary amine }\end{array}$ & $75 \%$ & $25 \%$ & 76 & 17 & 1.6 \\
\hline
\end{tabular}

* Characterized by ${ }^{1} \mathrm{H}-\mathrm{NMR}$.

** Weight average molecular weight.

*** Polydispersity index $=$ ratio of weight average to number average molecular weight. 
a)

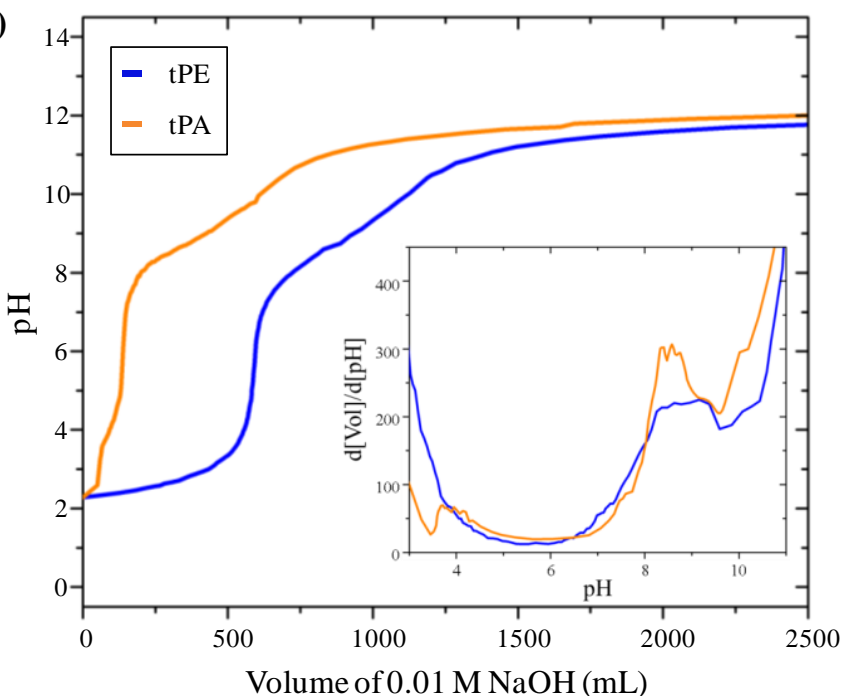

b)
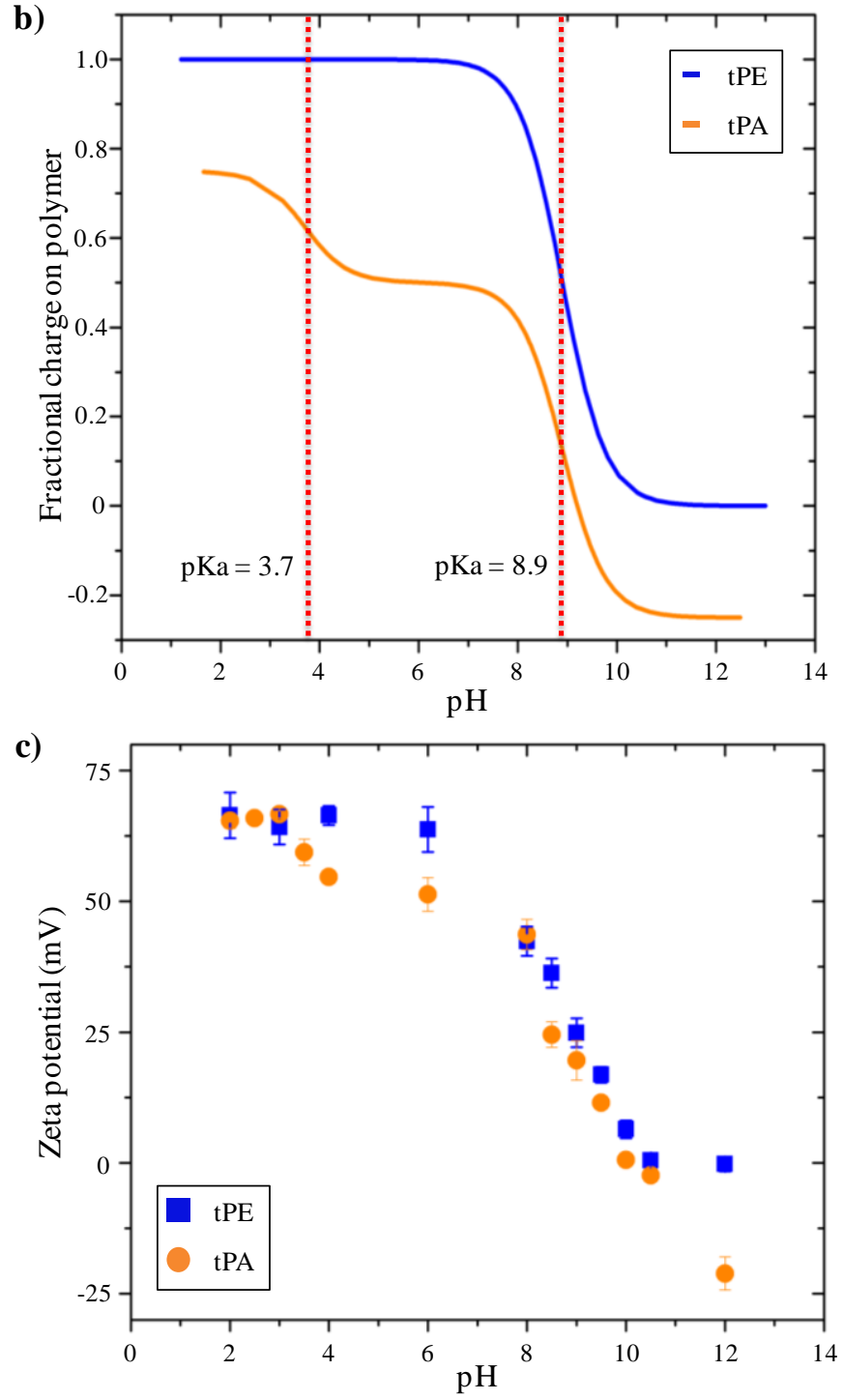
Figure 1. a) Titration curves of tPE (blue) and tPA (orange) flocculants, with the first derivative method (inset) applied to the raw titration curves. b) Fractional charge as a function of $\mathrm{pH}$ calculated for tPE (blue) and tPA (orange) flocculants. The red vertical lines indicate the pKa values of 3.7 and 8.9 measured from the titration data for the acrylic acid and DMAPAA components, respectively. c) Zeta potential values as a function of $\mathrm{pH}$ for the tPE (blue squares) and tPA (orange circles) flocculants. 
a)

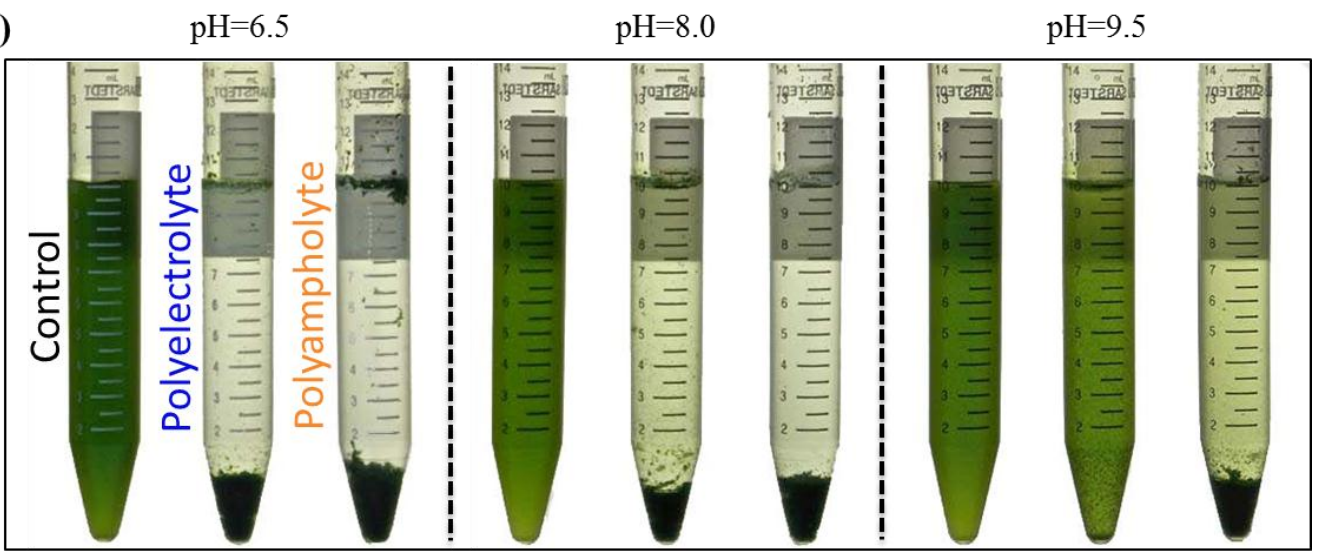

b)

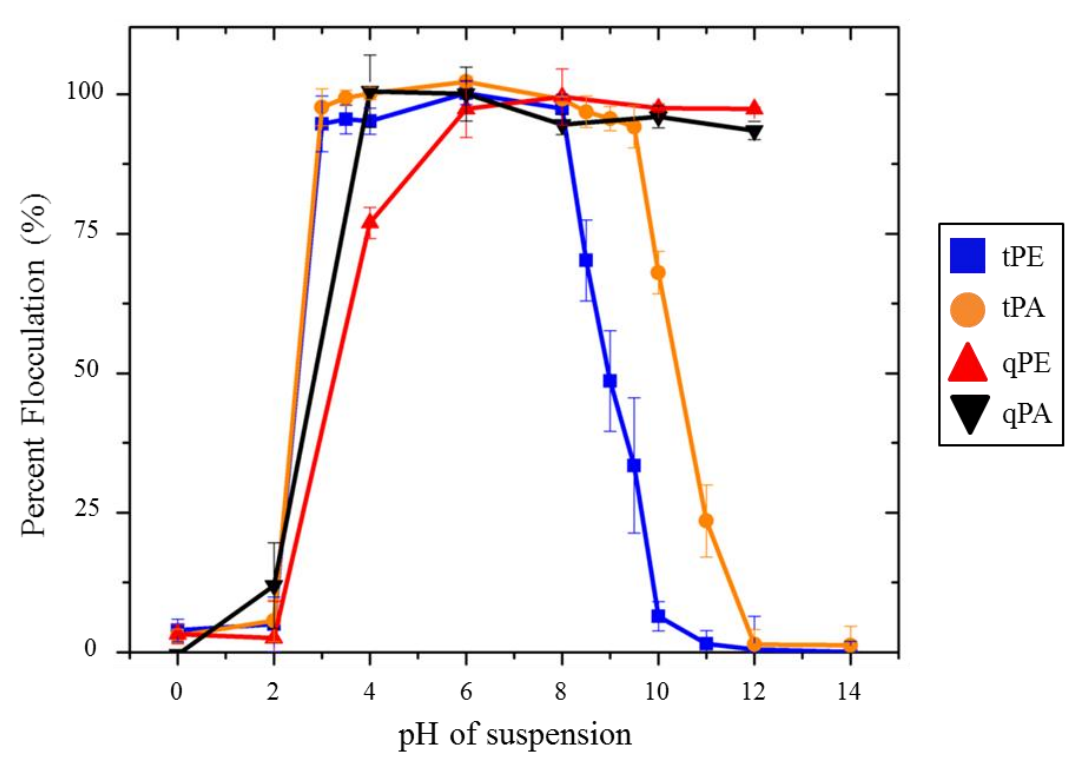

Figure 2. a) Chlorella vulgaris in $10 \mathrm{~mL}$ volumes under conditions of natural sedimentation (left, standard control without any polymer flocculant) and flocculated with tPE (middle) and tPA (right) flocculants at suspension $\mathrm{pH}$ values of 6.5, 8.0, and 9.5. b) Percent flocculation as a function of the $\mathrm{pH}$ of the suspension, including for flocculants with quaternary amine functionalities that are not $\mathrm{pH}$ sensitive (qPE and $\mathrm{qPA}$ ). The error bars represent one standard deviation of triplicate samples for each condition. 


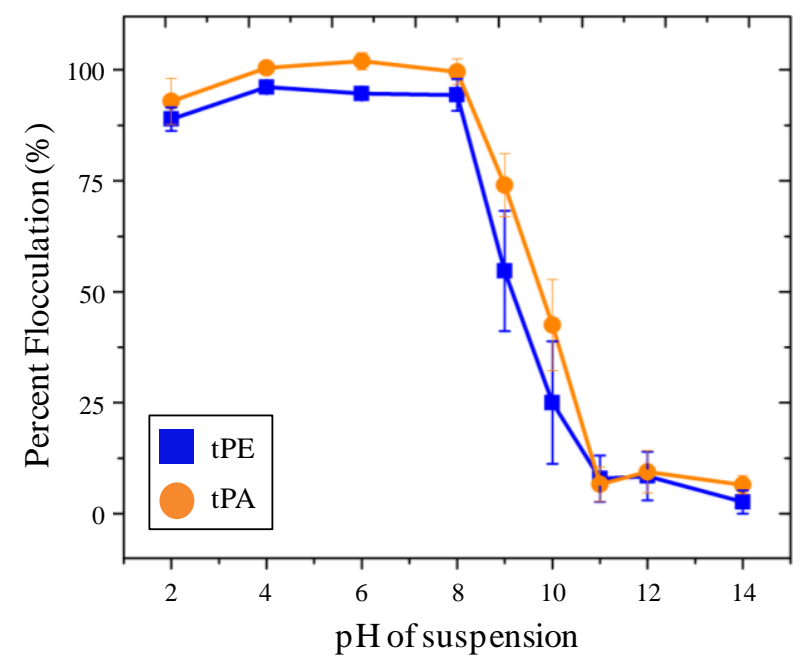

Figure 3. Percent flocculation of a solely negatively-charged colloidal suspension of silica microparticles as a function of suspension $\mathrm{pH}$. The error bars represent one standard deviation of the triplicate samples for each $\mathrm{pH}$ solution. 


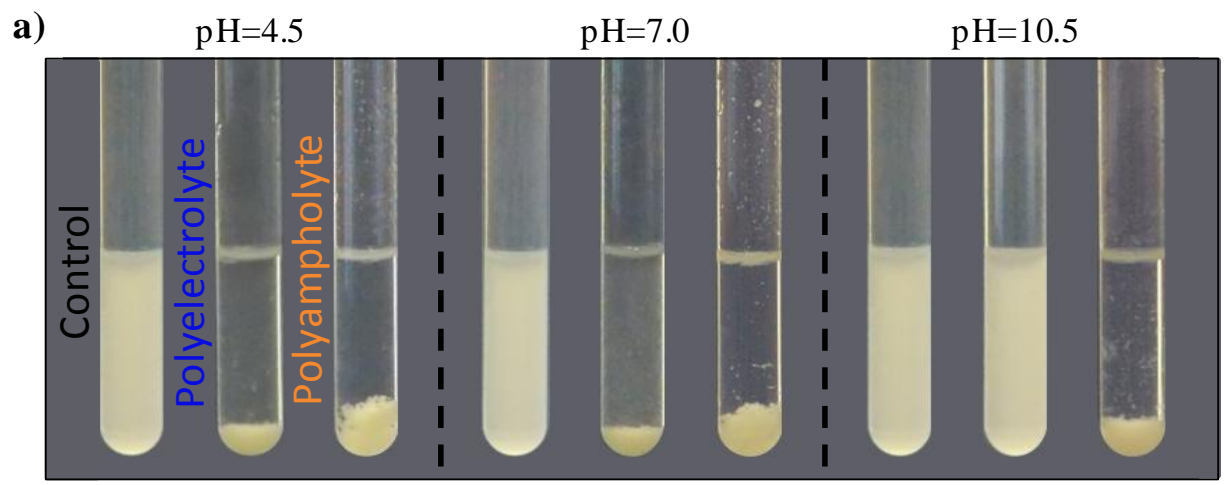

b)

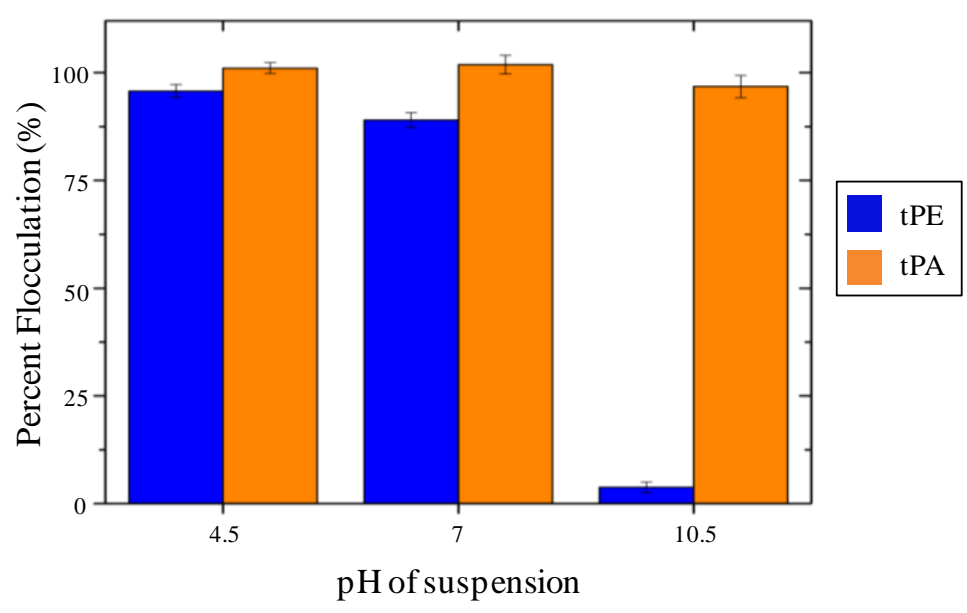

Figure 4. a) Saccharomyces cerevisiae in $4 \mathrm{~mL}$ volumes under conditions of natural sedimentation (left, standard control without any polymer flocculant) and flocculated with tPE (middle) and tPA (right) flocculants at suspension $\mathrm{pH}$ values of 4.5, 7.0, and 10.5. b) Percent flocculation as a function of the $\mathrm{pH}$ of the suspension. The error bars represent one standard deviation of triplicate samples for each condition. 
a)

$\mathrm{pH}=9.0$

$\mathrm{pH}=10.5$

$\mathrm{pH}=12.0$

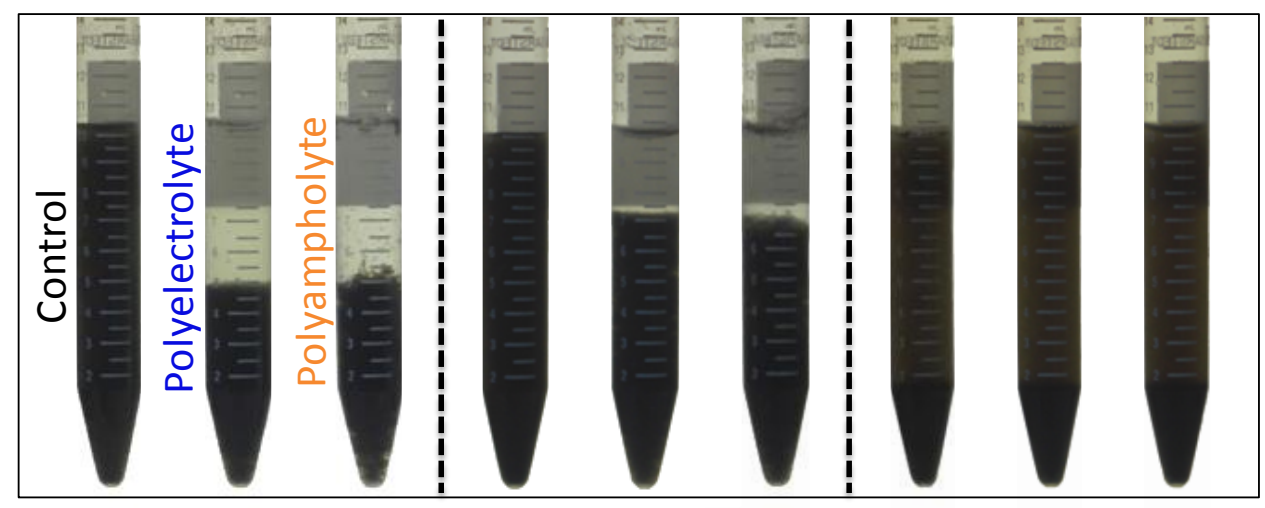

b)

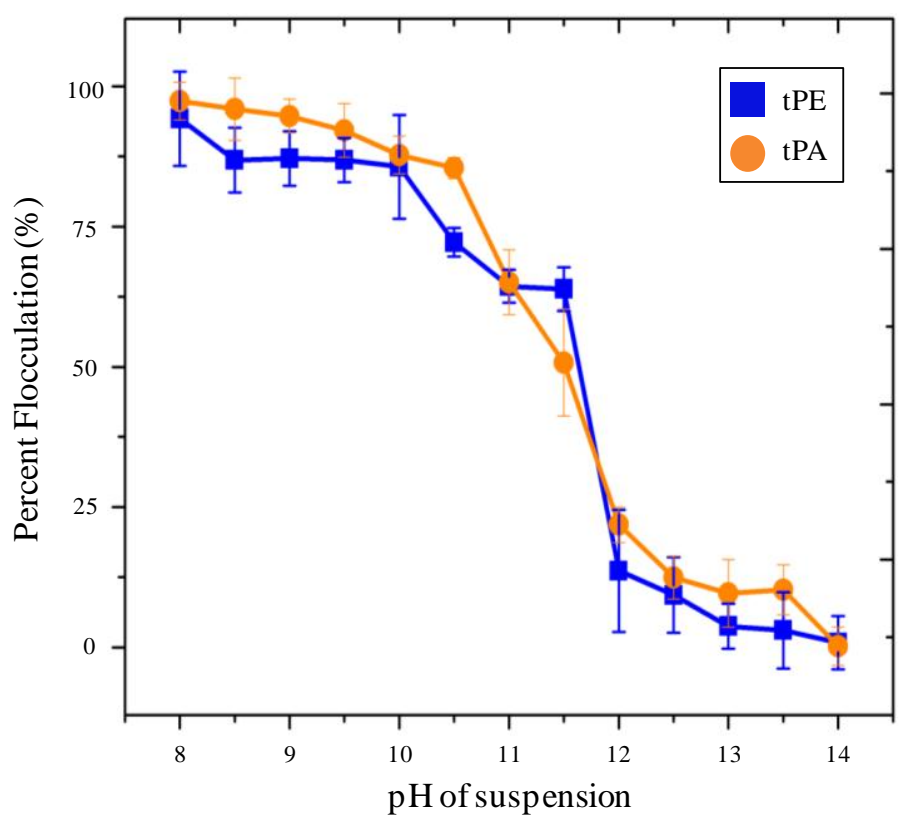

Figure 5. a) Wastewater sludge in $10 \mathrm{~mL}$ volumes under conditions of natural sedimentation (left, standard control without any polymer flocculant) and flocculated with tPE (middle) and tPA (right) flocculants at suspension $\mathrm{pH}$ values of 9.0, 10.5, and 12.0. b) Percent flocculation as a function of the $\mathrm{pH}$ of the suspension. The error bars represent one standard deviation of triplicate samples for each condition. 


\section{References:}

[1] A.A. Baran, Flocculation of cellular suspensions by polyelectrolytes, Colloids Surf. 31 (1988) 259-264.

[2] S.R. Wickramasinghe, Y.-K. Leong, S. Mondal, J.-L. Liow, Influence of cationic flocculant properties on the flocculation of yeast suspensions, Advanced Power Technology 21 (2010) 374-379.

[3] L. Brennan P. Owende, Biofuels from microalgae-A review of technologies for production, processing, and extraction of biofuels and co-products, Renew. Sustain. Energy Rev. 14 (2010) 557-577.

[4] T.M. Mata, A.A. Martins, N.S. Caetano, Microalgae for biodiesel production and other applications: A review, Renew. Sustain. Energy Rev. 14 (2010) 217-232.

[5] G. Schaub, A. Vetter, Biofuels for automobiles - An overview, Chem. Eng. Technol. 31 (2008) 721-729.

[6] S. Barany, A. Szepesszentgyorgyi, Flocculation of cellular suspensions by polyelectrolytes, Adv. Colloid Interface Sci. 111 (2004) 117-129.

[7] L. Domingues, A.A. Vincente, N. Lima, J.A. Teixeira, Applications of yeast flocculation in biotechnological processes, Biotechnol. Bioprocess Eng. 5 (2000) 288-305.

[8] L. Eriksson, C. Axberg, Direct influence of wastewater pollutants on flocculation and sedimentation behaviour in biological wastewater treatment - I. Model system E. coli B, Water Research 15 (1980) 421-431.

[9] J. Pillai, Flocculants and coagulants: The keys to water and waste management in aggregate production. Illinois, Nalco Company (1997) 1-6p.

[10] A.A. Shukla, M.R. Etzel, S. Gadam, Process scale bioseparations for the biopharmaceutical industry, CRC Press, Boca Raton, FL, 2007.

[11] A.A. Shukla, C. Jiang, J. Ma, M. Rubacha, L. Flanburg, S.S. Lee, Demonstration of robust host cell protein clearance in biopharmaceutical downstream processes, Biotechnol. Progress 24 (2008) 615-622.

[12] C.C. Wang, C.W. Chang, C.P. Chu, D.J. Lee, B.-V. Chang, C.S. Liao, Producing hydrogen from wastewater sludge by Clostridium bifermentans, J. Biotechnol. 102 (2003) 83-92.

[13] O.S. Amuda, I.A. Amoo, Coagulation/flocculation process and sludge conditioning in beverage industrial wastewater treatment, J. Haz. Mater. 141(3) (2007) 778-783.

[14] N. Uduman, Y. Qi, M.K. Danquah, Dewatering of microalgal cultures: A major bottleneck to algae-based fuels, J. Renewable Sustainable Energy 2 (2010) 1-15

[15] P. Spolaore, P, C. Joannis-Cassan, E. Duran, A. Isambert, Commercial applications of microalgae, J. Bioscience and Bioeng. 101 (2006) 87-96.

[16] R. Pratt, Influence of the size of the growth of Chlorella vulgaris in freshly prepared culture medium, Am. J. Bot. 27 (1940) 52-56.

[17] G.A. Shelef, A. Sukenik, M. Green, Microalgae harvesting and processing: A literature review, Report SERI/STR-231-2396, Solar Energy Research Institute, Golden, CO, 1984. 
[18] I. Szilagyi, A. Sadeghpour, M. Borkovec, Destabilization of colloidal suspensions by multivalent ions and polyelectrolytes: From screening to overcharging, Langmuir 28 (2012) 6211-6215.

[19] K.B. Musabekov, N.K. Tusupbaev, S.E. Kudaibergenov, Interaction of synthetic polyampholytes with disperse particles, Macromol. Chem. Phys. 199(3) (1998) 401-408.

[20] M.W. Tenney, W.F. Echelberger, R.G. Schuessler, J.L. Pavoni, Algae flocculation with synthetic organic polyelectrolytes, Appl. Microbiol. 18 (1969) 965-971.

[21] S. Hadjoudja, V. Deluchat, M. Baudu, Cell surface characterization of Microcystis aeruginosa and Chlorella vulgaris, J. Colloid and Interface Science 342 (2010) 293-299.

[22] J.S. Baker, L.Y. Dudley, Biofouling in membrane systems - a review, Desalination 118 (1998) 81-89.

[23] H.C. Flemming, G. Schaule, T. Griebe, J. Schmitt, A. Tamachkiarowa, Biofouling - the Achilles heel of membrane processes, Desalination 113 (1997) 215-225.

[24] S. Babel, S. Takizawa, Microfiltration membrane fouling and cake behaviour during algal filtration, Desalination 261 (2010) 46-51.

[25] M.R. Bilad, D. Vandamme, I. Foubert, K. Muylaert, I.F.J Vankelecom, Harvesting microalgal biomass using submerged microfiltration membranes, Bioresource Technology 111 (2012) 343-352.

[26] D.A. Ladner, D.R. Vardonaand, M.M. Clark, Effects of shear on microfiltration and ultrafiltration fouling by marine bloom-forming algae, J. Membrane Science 356 (2010) 33-43.

[27] P. Blanpain-Avet, C. Faille, G. Delaplace, T. Bénézech, Cell adhesion and related fouling mechanism on a tubular ceramic microfiltration membrane using Bacillus cereus spores, J. Membrane Science 385-386 (2011) 200-216.

[28] Y. Zhang, Y. Zhao, H. Chu, X. Zhou, B. Dong, Dewatering of Chlorella pyrenoidosa using diatomite dynamic membrane: Filtration performance, membrane fouling and cake behavior, Colloids and Surfaces B: Biointerfaces 113 (2014) 458-466.

[29] J.-S. Kim, S. Akeprathumchai, S.R. Wickramasinghe, Flocculation to enhance microfiltration, J. Membrane Science 182 (2001) 161-172.

[30] S. Neyret, L. Ouali, F. Candau, E. Pefferkorn, Adsorption of polyampholytes on polystyrene latex: Effect on colloid stability, J. Colloid Interface Sci. 176 (1995) 86-94.

[31] S. Schwarz, W. Jaeger, B.R. Paulke, S. Bratskaya, N. Smolka, J. Bohrisch, Cationic flocculants carrying hydrophobic functionalities: Applications for solid/liquid separation, J. Phys. Chem. B. 111 (2007) 8649-8654.

[32] D.R. Kasper, Theoretical and experimental investigations of the flocculation of charged particles in aqueous solutions by polyelectrolytes of opposite charge, $\mathrm{PhD}$ Thesis, California Inst. Technol., Pasadena, CA, 1971.

[33] J. Gregory, The effect of cationic polymers on the colloidal stability of latex particles, J. Colloid Interface Sci. 55 (1976) 35-44.

[34] M. Dittrich, S. Sibler, Cell surface groups of two picocyanobacteria strains studies by zeta 
potential investigations, potentiometric titration, and infrared spectroscopy, J. Colloid Interface Sci. 286 (2005) 487-495.

[35] H. Hayashi, H. Seiki, S. Tsuneda, A. Hirata, H. Sasaki, Influence of growth phase on bacterial cell electrokinetic characteristics examines by soft particle electrophoresis theory, J. Colloid Interface Sci. 264 (2003) 565-568.

[36] P.R. Rutter, B. Vincent, K.C. Marshall, (Eds.) Microbial adhesion and aggregation, Springer-Verlag, New York, 1984, p. 21.

[37] J. Hughes, D.K. Ramsden, K.C. Symes, The flocculation of bacteria using cationic synthetic flocculants and chitosan, Biotechnology Techniques 4(1) (1990) 55-60.

[38] C.G. Golueke, W.J. Oswald, Surface properties and ion exchange in algae removal, J. Water Poll. Control Fed. 42 (1970) R304-R314.

[39] J.L. Gardea-Torresdey, M.K. Becker-Hapak, J.M. Hosea, D.W. Darnall, Effect of chemical modification of algal carboxyl groups on metal ion binding, Env. Sci. Technol. 24 (1990) 1372-1378.

[40] K.J. Ives, The significance of surface electric charge on algae in water purification, J. Biochem. Microbiol. Tech. Engineer. 1 (1959) 37-47.

[41] R. Henderson, S.A. Parson, B. Jefferson, The impact of algal properties and pre-oxidation on solid-liquid separation of algae, Water Research 42(8-9) (2008) 1827-1845.

[42] X. Zhang, Z. Jiang, L. Chen, A. Chou, H. Yan, Y.Y. Zuo, X. Zhang, Influence of cell properties on rheological characterization of microalgae suspensions, Bioresource Tech. 139 (2013) 209-213.

[43] M.A. Hubbe, O.J. Rojas, D.S. Argyropoulos, Y. Wang, J. Song, N. Sulic, T. Sezaki, Charge and the dry-strength performance of polyampholytes Part 2. Colloidal effects, Colloids and Surfaces A: Physicochem. Eng. Aspects 301 (2007) 23-32.

[44] G. Petzold, S. Schwarz, M. Mende, W. Jaeger, Dye flocculation using polyampholytes and polyelectrolyte-surfactant nanoparticles, J. Appl. Polym. Sci. 104 (2007) 1342-1349.

[45] Q. Zhang, D. Wang, Y. Bei, S. Ren, G. Fang, Flocculation performance of trimethyl quaternary ammonium salt of lignin-sodium alginate polyampholyte, BioResources 8 (2013) 3544-3555.

[46] T. Nonaka, H. Edawa, Studies of polymeric flocculants. XI. The preparation and properties of polyampholytes, Bull. Chem. Soc. Jpn. 53 (1980) 1632-1637.

[47] D. Bilanovic, G. Shelef, Flocculation of microalgae with cationic polymers - Effects of medium salinity, Biomass 17 (1988) 65-76.

[48] Z. Pan, Polymer Chemistry, Industry Press, Beijing, 2007.

[49] K.L. Morrissey, C. He, M.H. Wong, X. Zhao, R.Z. Chapman, S.L. Bender, W.D. Prevatt, M.P. Stoykovich, Charge-tunable polymers as reversible and recyclable flocculants for the dewatering of microalgae, Biotechnol. Bioeng. 112(1) (2015) 74-83.

[50] R.A. Anderson (Ed.), Algae Culturing Techniques, Elsevier, Boston, 2005.

[51] M.A. Borowitzka, L.J. Borowitzka (Eds.), Micro-algal Biotechnology, Cambridge 
University Press, Cambridge, 1988, pp. 357-394.

[52] K.L. Morrissey, M.I. Keirn, Y. Inaba, A.J. Denham, G.J. Henry, B.W. Vogler, M.C. Posewitz, M.P. Stoykovich, Recyclable polyampholyte flocculants for the cost-effective dewatering of microalgae and cyanobacteria, Algal Research 11 (2015) 304-312.

[53] M. Pavlovic, M. Adok-Sipiczki, C. Nardin, S. Pearson, E. Bourgeat-Lami, V. Prevot, I. Szilagyi, Effect of MacroRAFT copolymer adsorption on the colloidal stability of layered double hydroxide nanoparticles, Langmuir 31(46) (2015), 12609-12617.

[54] S. Hirzel, BUA report 197: Dimethyl aminopropylamine (DMAPA), GDCh - Advisory Committee on Existing Chemicals of Environmental Relevance (BUA), Wissenschaftliche Verlagsgesellschaft, 1998.

[55] C.A. Stales, S.R. Murphy, J.E. McLaughlin, H.W. Leung, T.C. Cascieri, C.H. Farr, Determination of selected fate and aquatic toxicity characteristics of acrylic acid and a series of acrylic esters, Chemosphere 40 (2000) 29-38.

[56] L.E. Svrdrup, T. Kallqvist, A.E. Kelley, S.B. Hagen, Comparative toxicity of acrylic acid to marine and freshwater microalgae and the significance for environmental effects assessments, Chemosphere 45 (2001) 653-658.

[57] G.V. Franks, Zeta potentials and yield stresses of silica suspensions in concentrated monovalent electrolytes: Isoelectric point shift and additional attraction, J. Colloid Interface Sci. 249 (2002) 44-51.

[58] This estimate assumes in part that the silica has a monodisperse particle diameter of $1 \mu \mathrm{m}$, that the silica particles are spherical and non-porous with a density of $2.196 \mathrm{~g} / \mathrm{cm}^{3}$, and that there is a surface charge density on the silica of 2000 negative charges $/ \mu \mathrm{m}^{2}$.

[59] D.E. Amory, P.G. Rouxhet, Surface properties of Saccharomyces cerevisiae and Saccharomyces carlsbergensis: Chemical composition, electrostatic charge and hydrophobicity, Biochimica et Biophysica Acta. 938 (1988) 61-70.

[60] A.A. Eddy, A.D. Rudin, The structure of the yeast cell wall. 1. Identification of charged groups at the surface, Proc. Royal Society Series B - Biological Sciences 148(932) (1958) 419-432.

[61] ANL, NREL, PNNL, Renewable Diesel from Algal Lipids: An Integrated Baseline for Cost, Emissions, and Resource Potential from a Harmonized Model, ANL/ESD/12-4; NREL/TP5100-55431; PNNL-21437, 2012.

[62] B.A. Kimball, E. Callender, E.V. Axtmann, Effects of colloids on metal transport in a river receiving acid mine drainage, upper Arkansas River, Colorado, USA, App. Geochem. 10 (1995) 285-306. 


\section{Statement of significance:}

This article presents the design and characterization of polyamphoteric flocculants for the enhanced separations of biocolloidal suspensions. The polyamphoteric flocculants consist of tunable, mixed charges dependent upon system $\mathrm{pH}$, thereby providing strong electrostatic attraction to the diversely-charged cellular surfaces of biological suspensions. Enhanced flocculation efficiencies are achieved, as compared to cationic polyelectrolyte flocculants, and result from the ability of polyampholytes to adsorb to a diverse range of charge character and operate over an extended range of $\mathrm{pH}$ values.

\section{Graphical abstract:}

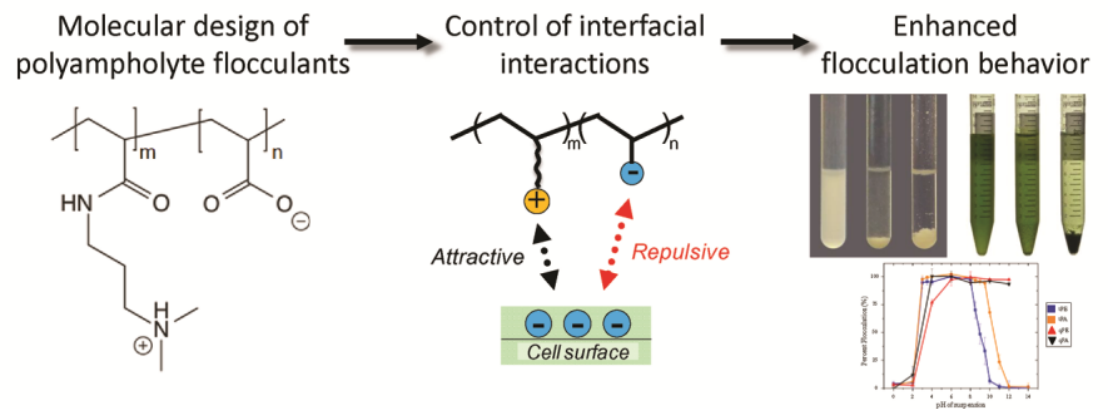

